\title{
Corrosion inhibition of $\alpha, \beta$-unsaturated carbonyl compounds on steel in acid medium
}

\author{
Gao Jiancun ${ }^{1,2}$, Weng Yongji ${ }^{*}$, Salitanate ${ }^{1,2}$, Feng $\mathrm{Li}^{2}$ and Yue Hong ${ }^{2}$ \\ ${ }^{1}$ China University of Petroleum (Beijing), Beijing 102249, China \\ ${ }^{2}$ Xinjiang Petroleum Institute, Urumqi, Xinjiang 830000, China
}

\begin{abstract}
Corrosion inhibition of three $\alpha, \beta$-unsaturated carbonyl compounds on N80 steel at high temperature and in concentrated acid medium was evaluated, and the inhibition mechanism was investigated. The results proved that both cinnamaldehyde and benzalacetone had an evident anticorrosion effect and could reduce the corrosion of steel effectively in acid medium. $\alpha, \beta$-unsaturated carbonyl compounds with a benzene ring structure had good adsorption on steel surface. The experiments proved that polymerization of $\alpha, \beta$-unsaturated carbonyl compounds on the steel surface at a high temperature and in concentrated acid medium resulted in a good corrosion inhibiting effect, which was attributed to the structures of $\alpha, \beta$-unsaturated carbonyl compounds.
\end{abstract}

Key words: $\alpha, \beta$-unsaturated carbonyl compounds, corrosion inhibitor, adsorption, oilfield acidizing treatment

\section{Introduction}

During the study of synthesis and performance of Mannich base corrosion inhibitors for hydrochloric acidizing fluids, we found that a $\alpha, \beta$-unsaturated ketone existed in the mother liquid of one type of Mannich base corrosion inhibitor (Gao et al, 2007), and proved through experiments that $\alpha, \beta$-unsaturated ketone had good synergy with Mannich base in hydrochloric acid because it had a benzene ring and conjugated carbonyl and carbon-carbon double bonds, which were similar to the structure of cinnamaldehyde. Cinnamaldehyde has become a new inhibitor in recent years, and it has attracted attentions due to its advantages of high efficiency and low toxicity (Wang et al, 1992; Ling, 1997; Zhen and Huang, 2000). Cinnamaldehyde and its derivatives are inhibitors containing benzene rings, conjugated carbonyl and carbon-carbon double bonds and they are not harmful to the environment. Thereafter in this paper, cinnamaldehyde and similar structured benzalacetone and chalcone were used to study their inhibition mechanism.

\section{Experimental methods}

\subsection{Synthesis of $\alpha, \beta$-unsaturated carbonyl compounds}

Hypnone or benzaldehyde and $\mathrm{NaOH}$ solution were added to a 3-neck flask equipped with a magnetic stirrer, a thermometer, a dropping funnel and a ball condenser. formaldehyde or acetic aldehyde was dropped at a controlled speed into the flask and stirred for an hour while the reaction

*Corresponding author. email: wengyj@cup.edu.cn

Received September 8, 2008 temperature was controlled by a cold water bath. The reaction liquid was transferred to a separating funnel and the organic phase was separated. A $5 \% \mathrm{HCl}$ solution was added to the organic phase to adjust the $\mathrm{pH}$ to 7 and some anhydrous $\mathrm{MgSO}_{4}$ was added for drying. After filtration it was distilled under vacuum to distill out the hypnone or benzaldehyde (Liu, 2005; Yu and Jiang, 1999; Moorthy et al, 2006).

The synthesized $\alpha, \beta$-unsaturated carbonyl compounds included cinnamaldehyde, benzalacetone and chalcone, with the following reaction principle: under the action of dilute alkali solution $\alpha, \beta$-unsaturated carbonyl compounds were formed by condensation.

Cinnamaldehyde, benzylideneacetone, and chalcone

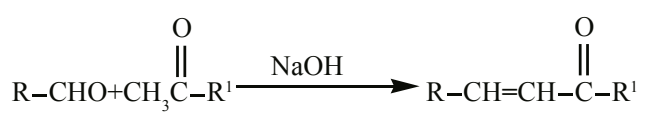

were synthesized by the methods reported in literatures (Liu, 2005; Yu and Jiang, 1999; Moorthy et al, 2006). Their structural formulas are as below:

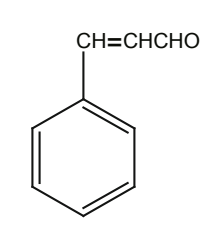

cinnamaldehyde

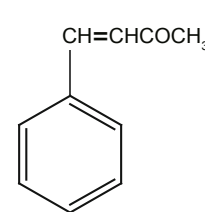

benzalacetone

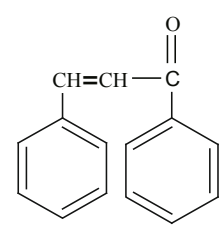

chalcone

\subsection{Infrared spectrum analysis and test of synthesized products}

(a) The synthesized products were identified with an FT-IR Bio-Rad FTS-165 spectrometer made by Bio-Rad 
Laboratories, Inc., USA, using the $\mathrm{KBr}$ disk method, from 4,000-400 $\mathrm{cm}^{-1}$.

(b) Physical adsorption IR test of the inhibitors: The inhibitor was coated evenly on the surface of the N80 steel test coupon and dried in air then the IR spectrum was collected on the same spectrometer.

(c) IR adsorption test after reaction of the inhibitors: The N80 steel test coupon was put in the solution ( $1 \%$ inhibitor in $20 \% \mathrm{HCl}$ solution) and heated to $90{ }^{\circ} \mathrm{C}$ for 0.5 hour. Then it was taken out and dried in air before IR test.

\subsection{Evaluation of inhibition effect by weight loss method}

The test coupons were polished with a series of metallographic abrasive paper and cleaned with acetone to remove oils. Then, the dimension and weight of the coupons were measured and they were cleaned in absolute alcohol and washed with distilled water. After that the coupons were hung completely immersed in a big test tube full of preheated acid. The experimental phenomena were observed and recorded. Four hours later the steel coupons were taken out and cleaned with acetone and absolute alcohol to remove the residues, cleaned with filter paper, dried with a blower and then weighed with an electronic balance, M-TAE100 made by Mettler-Toledo International Inc., with an accuracy of $0.0001 \mathrm{~g}$.

\subsection{Electrochemical method for investigation of inhibition mechanism}

A computer controlled potentiostat (CS300U/UA, Wuhan CorrTest Instrument Co. Ltd.) was used in the experiment. The experimental medium was $5 \% \mathrm{HCl}$ solution. The working electrode was a N80 steel mounted electrode with an exposed area of $0.5 \mathrm{~cm}^{2}$. A saturated calomel electrode (SCE) was used as reference electrode and platinum electrode was used as counter electrode. The scanning region was $-500 \mathrm{mV}$ to $500 \mathrm{mV}$, and the potential scanning rate was $5 \mathrm{mV} / \mathrm{s}$ (Tian 1984; Yang and Lu, 2001). Electrochemical polarization curves and differential polarization curves were obtained (Wang et al, 1999).

\section{Conclusions and discussion}

\subsection{Cinnamaldehyde}

\subsubsection{IR test of cinnamaldehyde}

Fig. 1 shows the infrared spectrogram of the synthesized c innamaldehyde.

Fig. 2 is the standard infrared spectrogram of cinnamaldehyde from Spectral Database for Organic Compounds (SDBS, this is a free site organized by National Institute of Advanced Industrial Science and Technology (AIST), Japan).

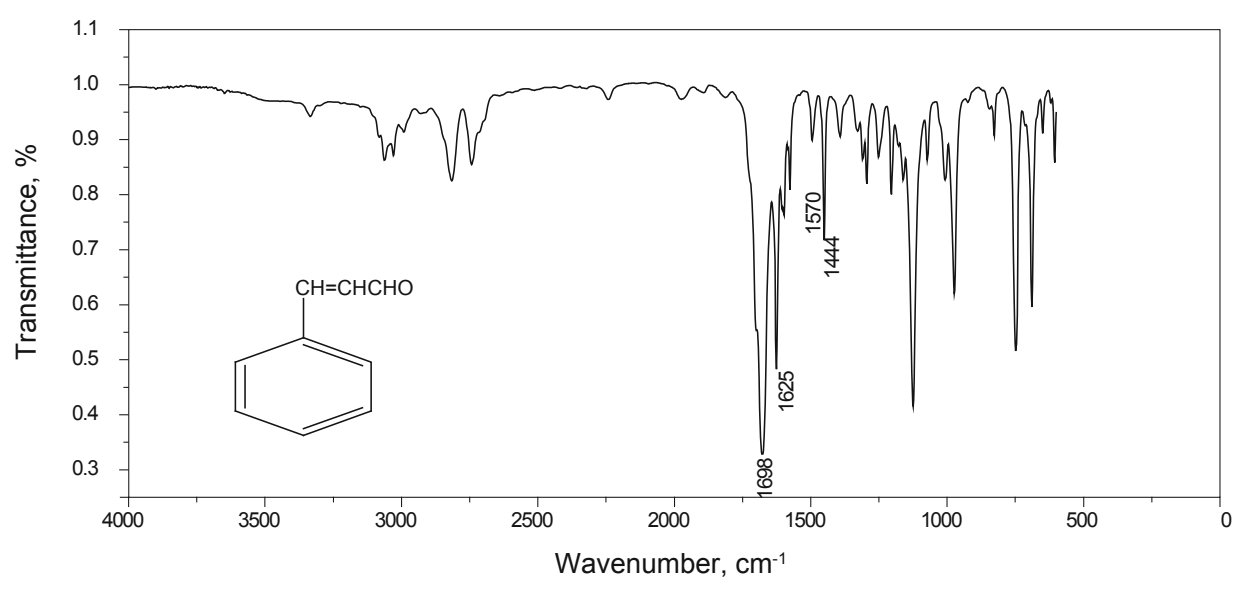

Fig. 1 Infrared absorption spectrum of the synthesized cinnamaldehyde

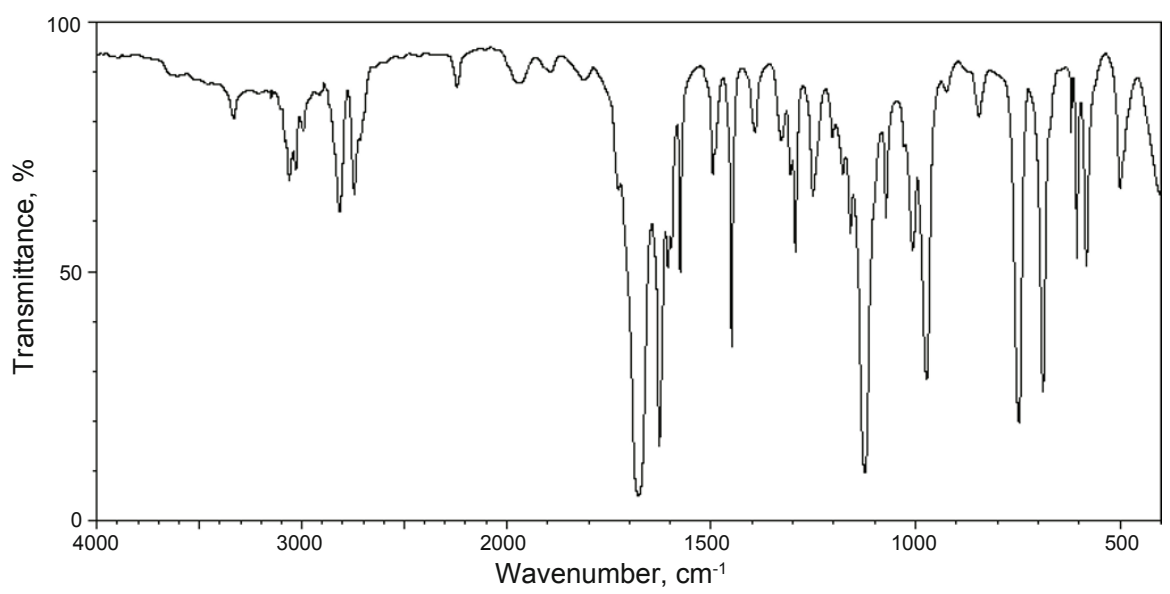

Fig. 2 Standard infrared absorption spectrum of cinnamaldehyde form SDBS 
Fig. 1 and Fig. 2 show that the main spectral peaks of the synthesized product are in accordance with the characteristic peaks of main functional groups of cinnamaldehyde such as $-\mathrm{C}=\mathrm{C},-\mathrm{C}=\mathrm{O}$ and benzene ring, proving that the synthesized product was cinnamaldehyde.

\subsubsection{IR adsorption test before and after reaction in $\mathrm{HCl}$ solution}

Fig. 3 is the IR absorption spectrum of cinnamaldehyde after it was coated on the N80 steel coupon and dried in air. Fig. 4 shows the infrared absorption spectrum of

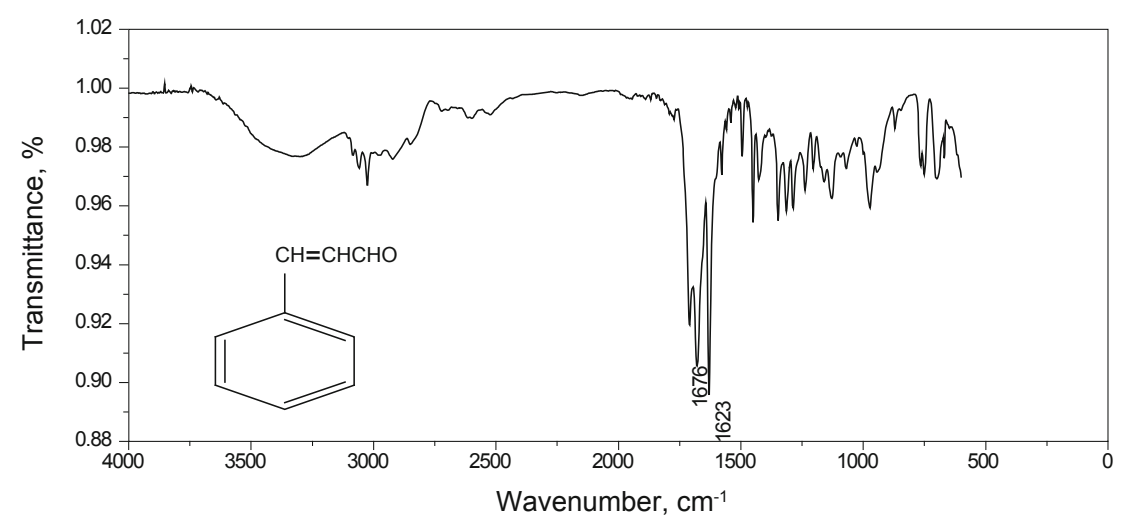

Fig. 3 Infrared absorption spectrum of cinnamaldehyde before reaction in $\mathrm{HCl}$ solution

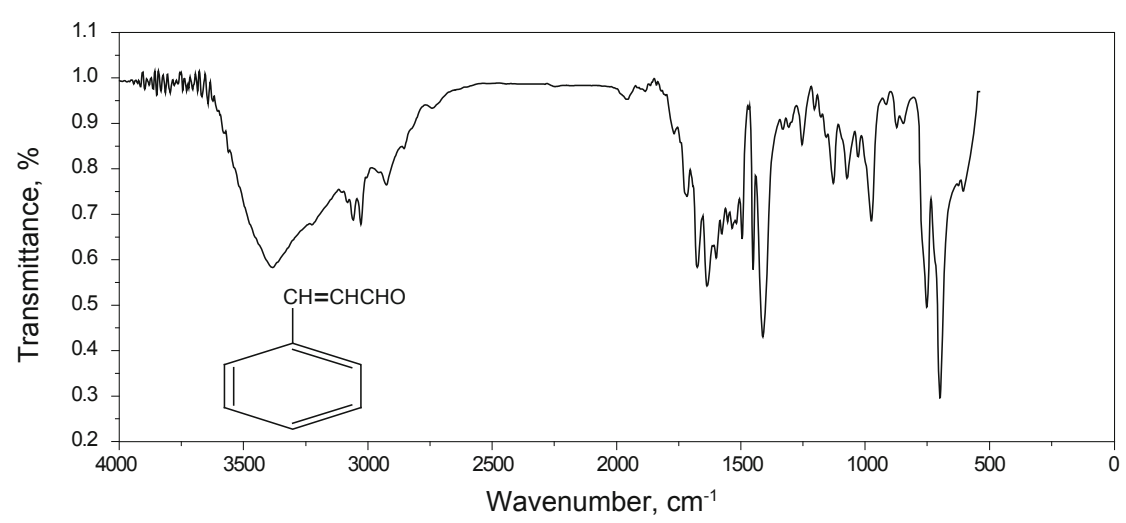

Fig. 4 Infrared absorption spectrogram of cinnamaldehyde after reaction with $20 \% \mathrm{HCl}$ solution for $0.5 \mathrm{~h}\left(90^{\circ} \mathrm{C}\right)$

cinnamaldehyde after it was adsorbed on the test coupon and immersed in $20 \% \mathrm{HCl}$ solution at $90^{\circ} \mathrm{C}$ for $0.5 \mathrm{~h}$.

Compared with the standard spectrogram in Fig. 2, the spectral characteristic peaks of the main functional groups of cinnamaldehyde in Fig. 3 do not change, indicating that only simple physical adsorption occurred. After reaction with $20 \% \mathrm{HCl}$ at $90{ }^{\circ} \mathrm{C}$ for 0.5 hour, a thin oil film was formed on the steel coupon. The IR spectrogram (Fig. 4) shows that the characteristic peaks of some main functional groups of cinnamaldehyde, such as $-\mathrm{C}=\mathrm{C},-\mathrm{C}=\mathrm{O}$, disappeared, implying that cinnamaldehyde polymerized to a bigger molecule, and the polymer was adsorbed on the surface of the steel, which may be the reason why cinnamaldehyde is good corrosion inhibit for steel in acid.

\subsection{Benzalacetone}

\subsubsection{IR test of benzalacetone}

Fig. 5 shows the infrared spectrogram of the synthesized

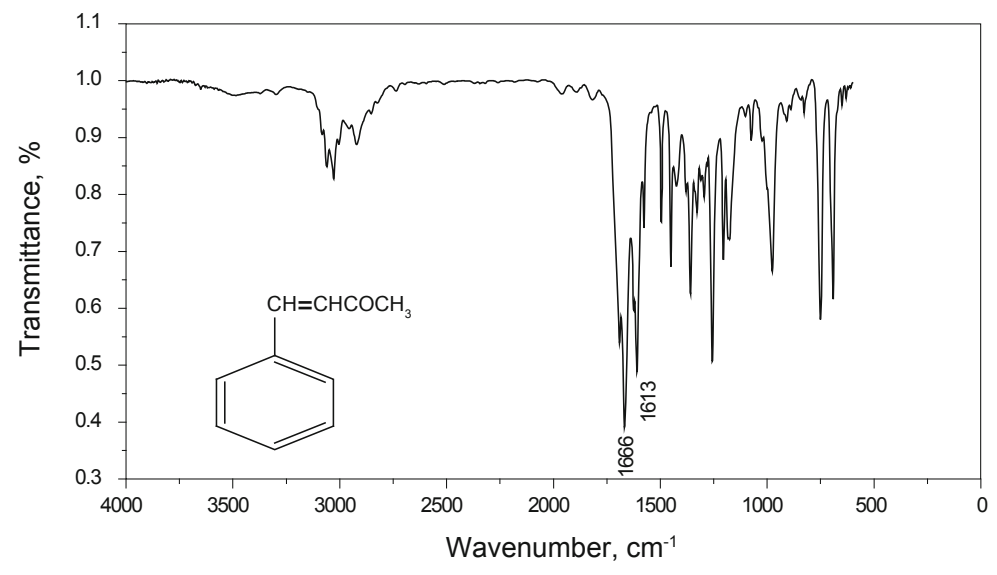

Fig. 5 Infrared absorption spectrum of the synthesized benzalacetone 


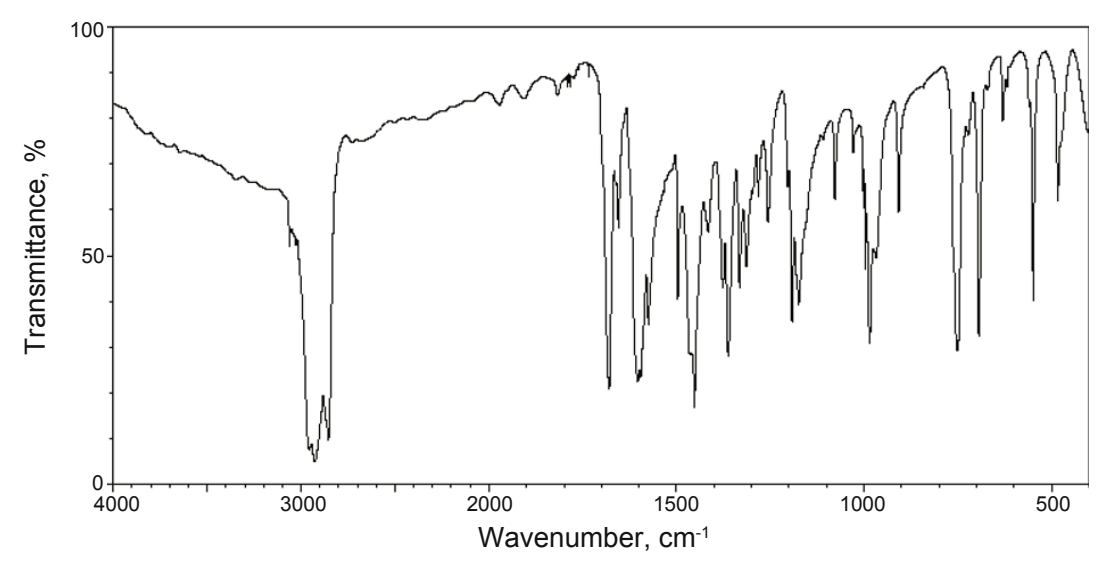

Fig. 6 Standard infrared absorption spectrum of benzalacetone form SDBS

benzalacetone. Fig. 6 is the standard infrared spectrogram of benzalacetone from SDBS.

Comparison of Fig. 5 and Fig. 6 indicates that the main spectral peaks of the synthesized product are in accordance with the characteristic peaks of the main functional groups of benzalacetone such as $-\mathrm{C}=\mathrm{C},-\mathrm{C}=\mathrm{O}$ and benzene ring, which proves that the synthesized product is benzalacetone.

\subsubsection{IR adsorption test before and after reaction in $\mathrm{HCl}$ solution}

Fig. 7 is the IR absorption spectrum of benzalacetone after it was coated on a steel coupon and dried in air. Fig. 8 shows the infrared absorption spectrum of benzalacetone after it was adsorbed on $\mathrm{N} 80$ steel and reacted with $20 \% \mathrm{HCl}$ solution at $90{ }^{\circ} \mathrm{C}$ for $0.5 \mathrm{~h}$.

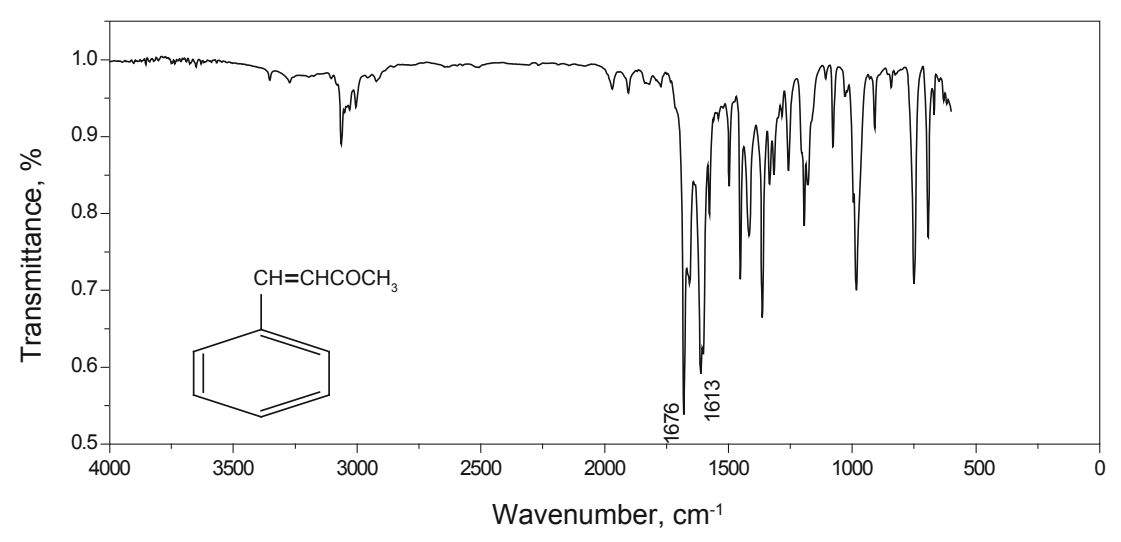

Fig. 7 Infrared absorption spectrum of benzalacetone before reaction in $\mathrm{HCl}$ solution

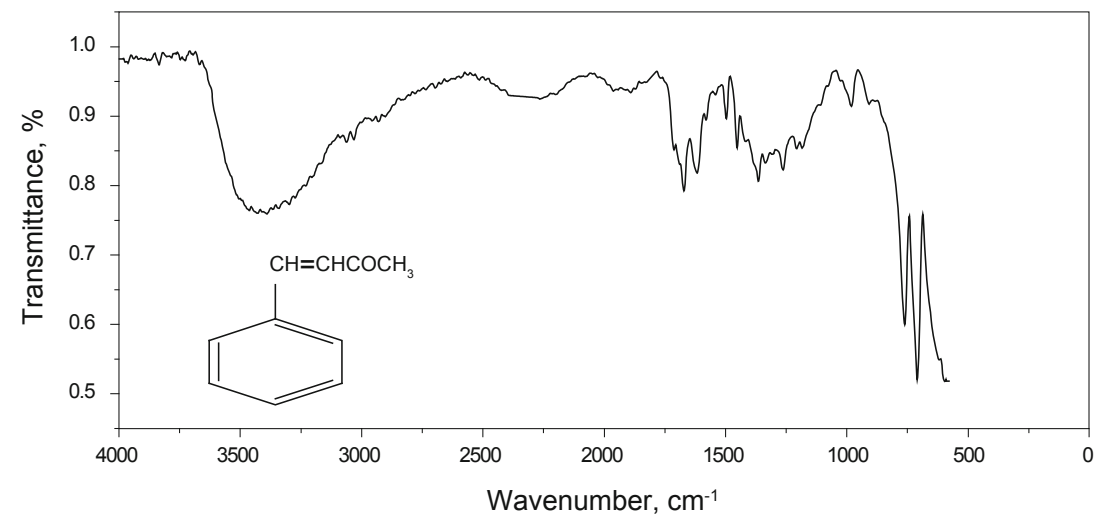

Fig. 8 Infrared absorption spectrum of benzalacetone after reaction with $20 \% \mathrm{HCl}$ solution for $0.5 \mathrm{~h}\left(90^{\circ} \mathrm{C}\right)$

The spectral characteristic peaks of the main functional groups of benzalacetone do not change in Fig.7, the same as shown in Fig. 5, but in Fig. 8, the characteristic peaks of the main functional group such as $-\mathrm{C}=\mathrm{C}$ and $-\mathrm{C}=\mathrm{O}$ have changed but not significantly, indicating that benzalacetone polymerized partially in $20 \% \mathrm{HCl}$ solution at $90{ }^{\circ} \mathrm{C}$ and the polymer was adsorbed on the surface of the steel coupon protecting it from corrosion.

\subsection{Chalcone}

\subsubsection{IR test of chalcone}

Fig. 9 shows the infrared spectrogram of the synthesized 


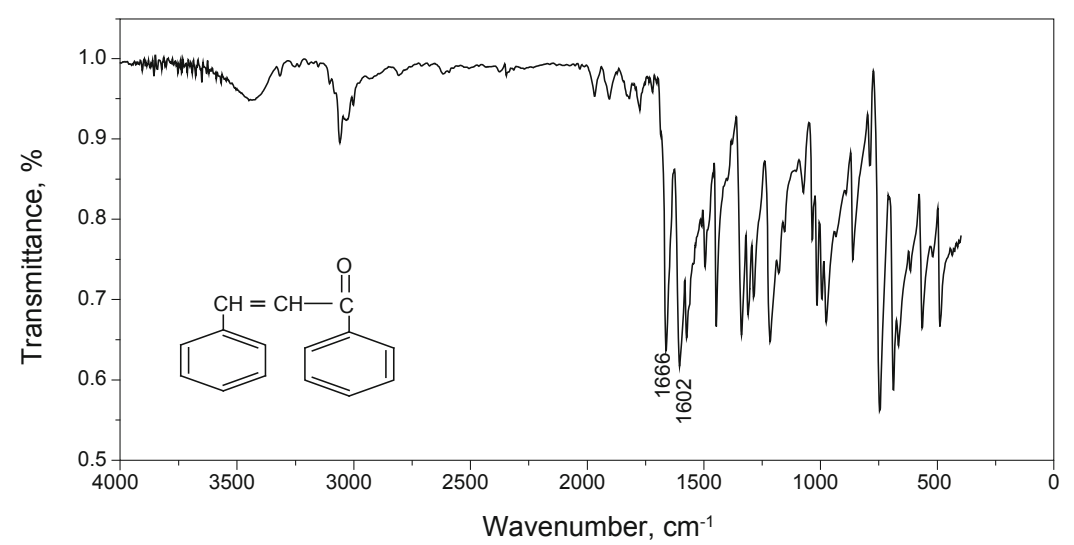

Fig. 9 Infrared absorption spectrum of the synthesized chalcone

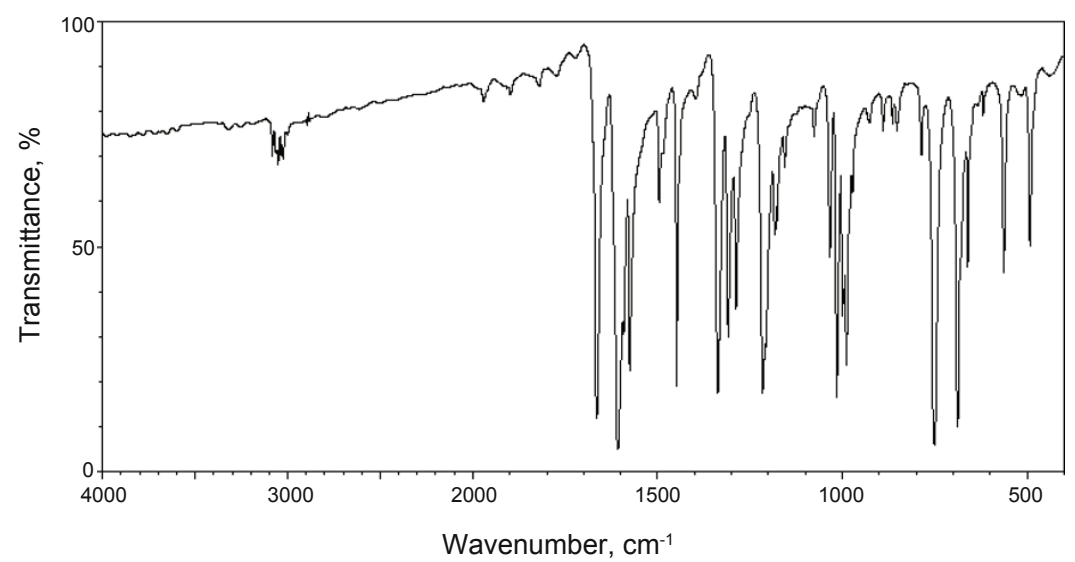

Fig. 10 Standard infrared absorption spectrum of chalcone form SDBS

chalcone. Fig. 10 is the standard infrared spectrogram of chalcone obtained from SDBS.

Comparison between Fig. 9 and Fig. 10 indicates that the main spectral characteristic peaks of the synthesized chalcone are in accordance with the spectral characteristic peaks of the main functional groups of chalcone such as $-\mathrm{C}=\mathrm{C}-,-\mathrm{C}=\mathrm{O}$ and benzene ring, which proves that the synthesized product is chalcone.

\subsubsection{IR adsorption test before and after chemical reaction in $\mathrm{HCl}$}

Comparing Fig. 11 and Fig. 12 indicates that the intensity of the spectral characteristic peaks became weaker after chalcone was processed in $20 \% \mathrm{HCl}$ solution at $90{ }^{\circ} \mathrm{C}$, but the locations of main spectral characteristic peaks did not change.
The spectral characteristic peaks (1645 and 1613) of $-\mathrm{C}=\mathrm{C}-$ and $-\mathrm{C}=\mathrm{O}$ in Fig. 12 are identical with those in Fig. 11 (1666 and 1602). Because of the existence of two benzene rings in the molecule, it is difficult for chalcone to polymerize.

\subsection{Discussions on evaluation by weight loss method}

Evaluation of inhibition performance of different corrosion inhibitors at different concentrations by the weight loss method in $20 \% \mathrm{HCl}$ at $90{ }^{\circ} \mathrm{C}$ is shown in Table 1 .

Table 1 shows that with the increase of concentration of corrosion inhibitors, the corrosion rate decreased. The inhibition performance of both cinnamaldehyde and benzalacetone was enhanced with the increase of concentration and their inhibition performance became better after working together

Table 1 Comparison of inhibition performance of different inhibitors

\begin{tabular}{|c|c|c|c|c|}
\hline \multirow{2}{*}{ Inhibitor } & \multicolumn{4}{|c|}{ Corrosion rate at different concentrations of inhibitor, $\mathrm{g} \cdot \mathrm{m}^{-2} \cdot \mathrm{h}^{-1}$} \\
\hline & $0.5 \mathrm{wt} \%$ & $1 w t \%$ & $\begin{array}{c}0.5 \mathrm{wt} \% \\
\text { (Containing } 5 \mathrm{wt} \% \text { propargyl alcohol) }\end{array}$ & $\begin{array}{c}1 \mathrm{wt} \% \\
\text { (Containing 10wt } \% \text { propargyl alcohol) }\end{array}$ \\
\hline Cinnamaldehyde & 22.0 & 6.3 & 8.4 & 2.9 \\
\hline Benzalacetone & 149.9 & 116.0 & 37.0 & 6.9 \\
\hline Chalcone & 633.0 & 412.5 & 65.4 & 14.7 \\
\hline
\end{tabular}




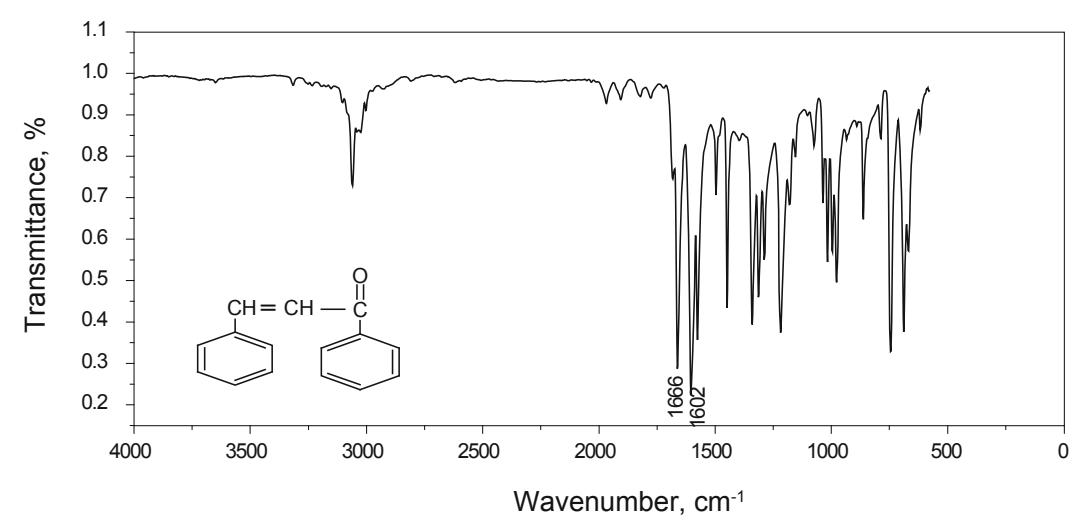

Fig. 11 Infrared absorption spectrum of chalcone on steel before reaction in $\mathrm{HCl}$ solution

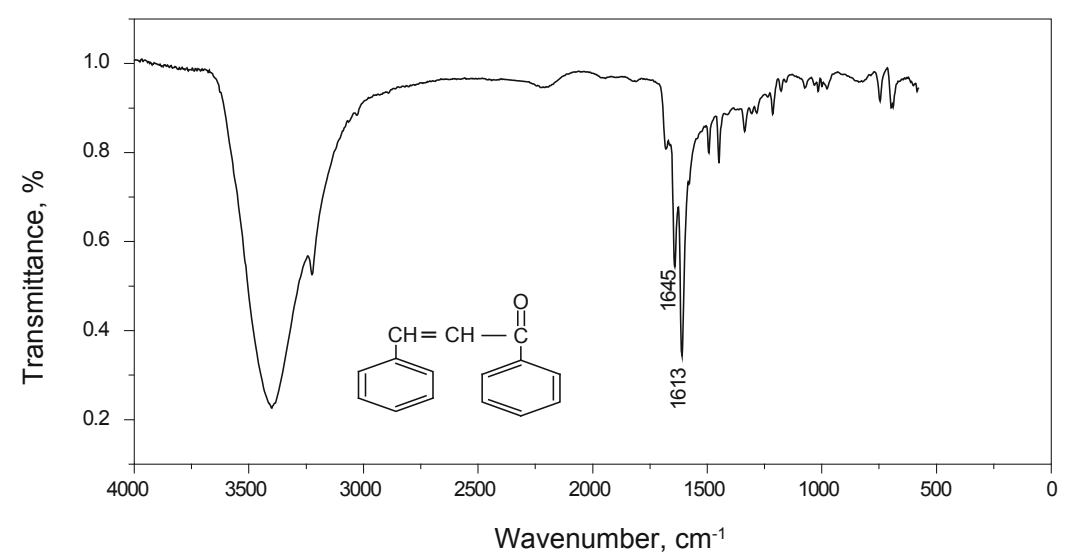

Fig. 12 Infrared absorption spectrum of chalcone on steel after reaction with $20 \% \mathrm{HCl}$ solution for $0.5 \mathrm{~h}\left(90^{\circ} \mathrm{C}\right)$

with propargyl alcohol. At the same concentration, the corrosion rate of cinnamaldehyde was the smallest, that of benzalacetone the next, and that of chalcone the largest. With the increase of inhibitor concentration, the difference became more significant, also proving that the inhibition effect of cinnamaldehyde was the best, benzalacetone the next, chalcone the worst.

\subsection{Discussions on electrochemistry test}

Potential scanning is a common method used in corrosion electrochemistry. Polarization curves obtained from potential scanning can be used to investigate the type of inhibitors and

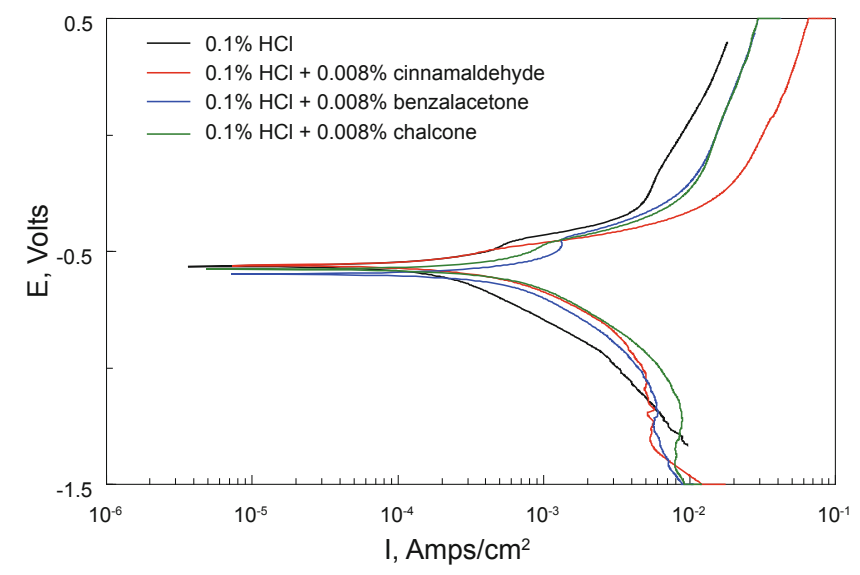

Fig. 13 Polarization curves at room temperature $\left(25^{\circ} \mathrm{C}\right)$ the mechanism of corrosion.

According to Fig. 13, at room temperature in $0.1 \% \mathrm{HCl}$ (in weight), the corrosion potentials of $\alpha, \beta$-unsaturated carbonyl compounds have not deviated, and both anodic reaction and cathodic reaction are restrained, so the inhibitors are the compound type. As shown in Fig. 14 and Fig. 15, in 15\% $\mathrm{HCl}$ solution at a higher temperature, it can be seen from the polarization curves of cinnamaldehyde and benzalacetone at different concentrations that corrosion potential have deviated upward significantly with increasing concentration and temperature, which implies that the inhibition to anode reaction $\mathrm{Fe}=\mathrm{Fe}^{2+}+2 \mathrm{e}$ has been reinforced .

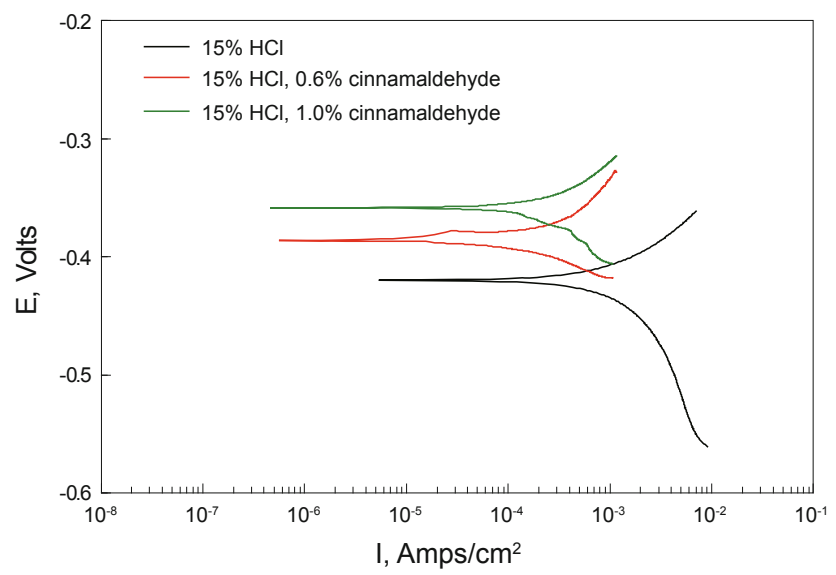

Fig. 14 Polarization curves of cinnamaldehyde at $70{ }^{\circ} \mathrm{C}$ 


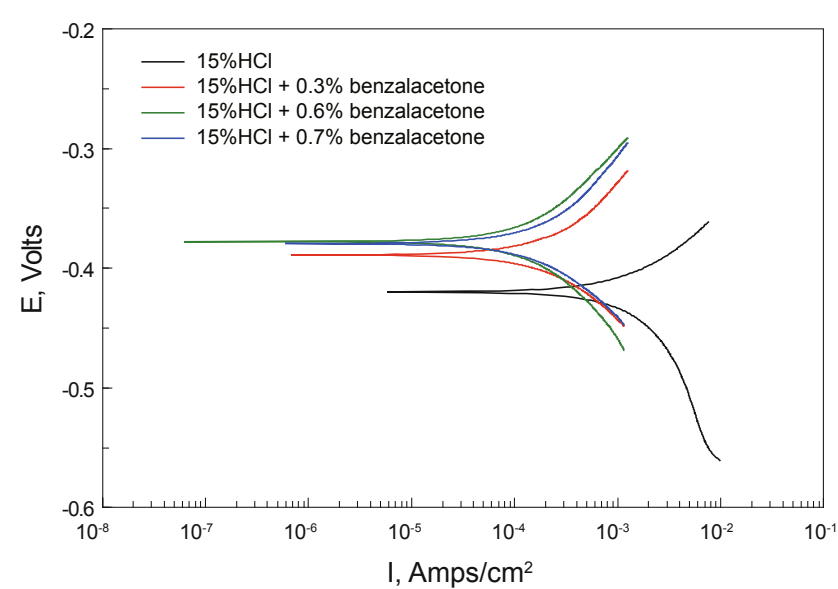

Fig. 15 Polarization curves of benzalacetone at $90{ }^{\circ} \mathrm{C}$

Through a differential treatment of Fig.13 with the definition of $\mathrm{D}=\mathrm{d} \operatorname{logi} / \mathrm{dE}$, i.e., reciprocal of semi-log graph of polarization curve, and plotting the D-E curve, the differential polarization curves of different inhibitors at room temperature can be obtained. After such conversion the current plateau in the original curve (Fig. 13) is now displayed as the desorption peak in the differential curve (Fig. 16). The differential curve proved that the surface coverage and adsorption stability of cinnamaldehyde on the surface of N80 steel are superior to those of benzalacetone and chalcone, which confirmed the conclusions of infrared spectrum analysis.

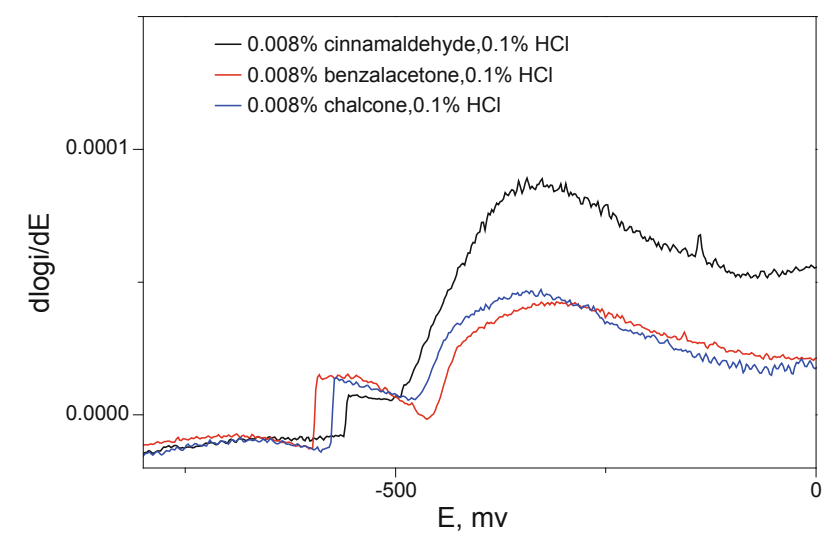

Fig. 16 Differential polarization curve at room temperature $\left(25^{\circ} \mathrm{C}\right)$

\section{Conclusions}

1) Cinnamaldehyde and benzalacetone protect steel effectively from corrosion at high temperature and in concentrated acid and greatly reduce the corrosion of steel.

2) $\alpha, \beta$-unsaturated carbonyl compounds with benzene structure had good adsorption on a steel surface in concentrated acid and their adsorption capacity was related to their molecular structures. Two benzene rings in chalcone molecule hindered chalcone from adsorption and reaction on steel surface.

3) Polymerization and adsorption on the surface of steel was the main reason for $\alpha, \beta$-unsaturated carbonyl compounds to be good corrosion inhibitors at high temperature in concentrated acid medium.

\section{References}

Gao J C, Feng L, Weng Y J, et al. Synergistic effect of Mannich base corrosion inhibition. Xinjiang Oil \& Gas. 2007. 3 (3): 86-88 (in Chinese)

Ling H C. Progress in Research of Inhibitors. Corrosion Science and Protection Technique. 1997. 4 (19): 308-313 (in Chinese)

Liu X M. Synthesis of Cinnarnaldehyde. Journal of Qufu Normal University. 2005. 2 (31): 96-98 (in Chinese)

Moorthy N S, Singh R J, Singh H P, et al. Synthesis biological evaluation and in silico metabolic and toxicity prediction of some fiavanone derivatives. Chem. Pharm. Bull. 2006. 54 (10): 13841390 (in English)

Tian S W. Electrochemistry Research Technique. Science Publishing House, 1984. 101-105 (in Chinese), Beijing

Wang D H, Bu X Z, Gan F X, et al. The Joint Adsorption/ Desorption of Organic Inhibitor and I' Anodic on Iron Electrode in Sulfuric Acid Solution. Corrosion Science and Protection Technolgy.1999.11 (1): 15-20 (in Chinese)

Wang J, Cao C N and Chen J J. The Recent Progress in the Theory and the Approach for the Study of Inhibitors. Corrosion Science and Protection Technique. 1992. 2 (14): 79-87 (in Chinese)

Yang H and Lu W Q. Applied electrochemistry. Chemical Industry Publishing House, 2001. 58 (in Chinese), Beijing

Yu S X and Jiang Y F. A Study On Synthetic Condition Of Benzalacetone. Journal of Gansu Education College (Natural Science). 1999. 1 (13): 46-48 (in Chinese)

Zhen J S and Huang Y K. Review and Prospect of Corrosion Inhibitor Development. Materials Protection. 2000. 5 (33): 11-15 (in Chinese)

(Edited by Zhu Xiuqin) 